\title{
Sharp Bounds of the Hyper-Zagreb Index on Acyclic, Unicylic, and Bicyclic Graphs
}

\author{
Wei Gao, ${ }^{1}$ Muhammad Kamran Jamil, ${ }^{2}$ Aisha Javed, ${ }^{3}$ Mohammad Reza Farahani, ${ }^{4}$ \\ Shaohui Wang, ${ }^{5}$ and Jia-Bao Liu ${ }^{6}$ \\ ${ }^{1}$ School of Information Science and Technology, Yunnan Normal University, Kunming 650500, China \\ ${ }^{2}$ Riphah Institute of Computing and Applied Sciences (RICAS), Riphah International University, Lahore, Pakistan \\ ${ }^{3}$ Abdus Salam School of Mathematical Sciences, Government College University, Lahore, Pakistan \\ ${ }^{4}$ Department of Applied Mathematics, Iran University of Science and Technology, Narmak, Tehran, Iran \\ ${ }^{5}$ Department of Mathematics and Computer Science, Adelphi University, Garden City, NY 11530, USA \\ ${ }^{6}$ School of Mathematics and Physics, Anhui Jianzhu University, Hefei 230601, China
}

Correspondence should be addressed to Jia-Bao Liu; liujiabaoad@163.com

Received 19 September 2016; Revised 16 December 2016; Accepted 27 December 2016; Published 1 February 2017

Academic Editor: J. R. Torregrosa

Copyright (c) 2017 Wei Gao et al. This is an open access article distributed under the Creative Commons Attribution License, which permits unrestricted use, distribution, and reproduction in any medium, provided the original work is properly cited.

\begin{abstract}
The hyper-Zagreb index is an important branch in the Zagreb indices family, which is defined as $\sum_{u v \in E(G)}(d(u)+d(v))^{2}$, where $d(v)$ is the degree of the vertex $v$ in a graph $G=(V(G), E(G))$. In this paper, the monotonicity of the hyper-Zagreb index under some graph transformations was studied. Using these nice mathematical properties, the extremal graphs among $n$-vertex trees (acyclic), unicyclic, and bicyclic graphs are determined for hyper-Zagreb index. Furthermore, the sharp upper and lower bounds on the hyper-Zagreb index of these graphs are provided.
\end{abstract}

\section{Introduction}

A topological index $\operatorname{Top}(G)$ of a graph $G$ is a number with this property that, for every graph $H$ isomorphic to $G$, $\operatorname{Top}(H)=$ $\operatorname{Top}(G)$. In 1947, Wiener determined the most widely known topological descriptor, the Wiener index [1]. He used it to determine physical properties of paraffin. The Wiener index of a graph is equal to the sum of distances between all pairs of vertices of related graphs. Numerous indices have been explored. The Zagreb indices are the most important topological indices, introduced by Gutman and Trinajstić more than thirty years ago [2]. For a graph $G=(V, E)$, the first and second Zagreb indices, $M_{1}$ and $M_{2}$, respectively, are defined as

$$
\begin{aligned}
& M_{1}(G)=\sum_{v \in V} d(v)^{2}, \\
& M_{2}(G)=\sum_{u v \in E} d(u) d(v) .
\end{aligned}
$$

In 1972, Zagreb indices first appeared in the topological formulas for the total $\pi$-energy of conjugated molecules [2]. For applications in QSPR/QSAR, latest results are referred to [3-8].

In 2004, Miličević et al. [9] reformulated Zagreb indices in terms of edge-degrees instead of vertex-degree as follows:

$$
\begin{aligned}
& E M_{1}(G)=\sum_{e \in E} d(e)^{2}, \\
& E M_{2}(G)=\sum_{e \sim f} d(e) d(f),
\end{aligned}
$$

where $d(e)$ is the degree of the edge $e$ in $G$, defined by $d(e)=$ $d(u)+d(v)-2$ with $e=u v$, and $e \sim f$ means that the edges $e$ and $f$ are adjacent. Some results related to $E M_{1}(G)$ and $E M_{2}(G)$ are given in [10-12]. 


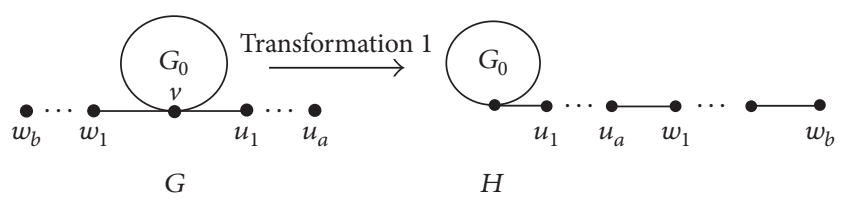

FIgURE 1: Two graphs.

In 2013, Shirdel et al. [13] introduced a new degree-based topological index named hyper-Zagreb index as

$$
H M(G)=\sum_{u v \in E}(d(u)+d(v))^{2} .
$$

Recently, the multiplicative versions of Zagreb indices are studied well in [14]. Motivated by these results [15-18], we explore the properties for the hyper-Zagreb index.

Let $G$ be a simple and connected graph with vertex set $V$ and edge set $E$. For a vertex $v \in V, N_{G}(v)$ denotes the set of all neighbors of $v$ in $G$. In a graph $G$, the number of independent cycles is called its cyclomatic number and is equal to $\gamma=m-$ $n+1$. Recall that graphs with $\gamma=0,1,2$ are referred to as trees, unicyclic graphs, and bicyclic graphs, respectively. $S_{n}, P_{n}$, and $C_{n}$ denote the star, path, and cycle on $n$ vertices, respectively. Let $v \in V$, and then let $G-v$ be a subgraph of $G$ by deleting vertex $v$ and adjacent edges. For $e \in E$, let $G-e$ be a subgraph of $G$ by deleting an edge $e$. Let $G$ be a nontrivial graph and let $v$ be its vertex. If $K$ is obtained from $G$ by fusing a tree $T$ at $v$, then we say that $T$ is a subtree of $K$ and $u$ is its root. The fusions of two vertices $u$ and $v$ in $G$ are denoted by $u+$ $v$. In order to exhibit our results, we introduce some graphtheoretical notations and terminology. For other undefined ones, see the book [19].

In this paper, we characterize the extremal properties of the hyper-Zagreb index. In Section 2, we present some graph transformations which increase or decrease $H M$. In Section 3, we determine the extremal acyclic, unicyclic, and bicyclic graphs with maximum and minimum hyper-Zagreb index.

\section{Graph Transformations}

In this section, we will introduce some graph transformations, which increase or decrease the hyper-Zagreb index. These transformations will help to prove our main results. The following one from $G$ to $H$ strictly decreases the hyperZagreb index of a graph.

Transformation 1. Let $G$ be a nontrivial connected graph and $v$ is a given vertex in $G$. Let $H$ be a graph obtained from $G$ by attaching two paths: $P_{1}=v u_{1} u_{2} \cdots u_{a}$ of length $a$ and $P_{2}=$ $v w_{1} w_{2} \cdots w_{b}$ of length $b$. If $H=G-v w_{1}+u_{a} w_{1}$, we say that $H$ is obtained from $G$ by Transformation 1, as shown in Figure 1.

Lemma 1. If $H$ is obtained from $G$ by Transformation 1 as shown in Figure 1, then

$$
H M(H)<H M(G) \text {. }
$$

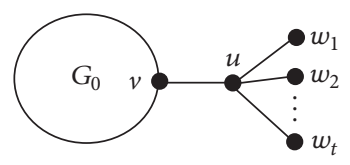

G

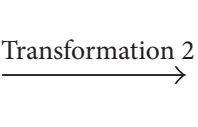

FiguRE 2: Transformation 2.

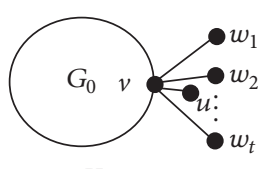

K
Proof. In applying Transformation 1 , the degree of $v$ decreases and the degrees of all its neighbors remain unchanged. So,

$$
\begin{aligned}
H M(G)-H M(H)> & \left(d_{G}(v)+d_{G}\left(w_{1}\right)\right)^{2} \\
& +\left(d_{G}(v)+d_{G}\left(u_{1}\right)\right)^{2} \\
& +\left(d_{G}\left(u_{k}\right)+d_{G}\left(u_{k-1}\right)\right)^{2} \\
& -\left(d_{G}(v)-1+d_{G}\left(u_{1}\right)\right)^{2} \\
& -\left(d_{G}\left(u_{k}\right)+1+d_{G}\left(u_{k-1}\right)\right)^{2} \\
& +\left(d_{K}\left(u_{k}\right)+d_{G}\left(w_{1}\right)\right)^{2} \\
= & \left(d_{G}(v)+2\right)^{2}+\left(d_{G}(v)+2\right)^{2} \\
& +3^{2}-\left(d_{G}(v)+1\right)^{2}-4^{2} \\
= & d_{G}(v)^{2}+6 d_{G}(v)>0 .
\end{aligned}
$$

Transformation 2. Let $u v$ be an edge of connected graph $G$ with $d_{G}(v) \geq 2$. Suppose that $\left\{v, w_{1}, w_{2}, \ldots, w_{t}\right\}$ are all the neighbors of vertex $u$, while $w_{1}, w_{2}, \ldots, w_{t}$ are pendant vertices. If $K=G-\left\{u w_{1}, u w_{2}, \ldots, u w_{t}\right\}+\left\{v w_{1}, v w_{2}, \ldots, v w_{t}\right\}$, we say that $K$ is obtained from $G$ by Transformation 2 , as shown in Figure 2.

Transformation 2 from $G$ to $K$ strictly increases $H M$ of a graph.

Lemma 2. If $K$ is obtained from $G$ by Transformation 2 as shown in Figure 2, then

$$
H M(G)<H M(K) \text {. }
$$

Proof. Clearly, $d_{G}(v)<d_{K}(v)$ and $(d(u)+d(v))$ is not changed during Transformation 2. Hence,

$$
\begin{aligned}
H M(K)-H M(G)> & (t+1)\left(d_{G}(v)+t+1\right)^{2} \\
& -\left(d_{G}(v)+d_{G}(u)\right)^{2} \\
& -t\left(d_{G}(u)+1\right)^{2} \\
= & (t+1)\left(d_{G}(v)+t+1\right)^{2} \\
& -\left(d_{G}(v)+t+1\right)^{2}-t(t+2)^{2} \\
= & t\left(d_{G}(v)+t+1\right)^{2}-t(t+2)^{2} \\
> & 0 .
\end{aligned}
$$




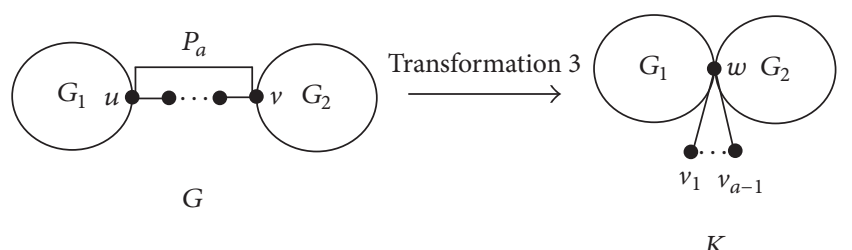

FIGURE 3: Transformation 3.
Transformation 3. Let $G$ be nontrivial connected graph and $u, v \in V(G)$. Let $P_{a}=(u=) v_{1} v_{2} \cdots v_{a}(=v)$ be a nontrivial $a$ length path of $G$ connecting vertices $u$ and $v$. If $K=G-$ $\left\{v_{1} v_{2}, v_{2} v_{3}, \ldots, v_{a-1} v_{a}\right\}+\left\{(u+v=) w v_{1}, w v_{2}, \ldots, w v_{a}\right\}$, we say that $K$ is obtained from $G$ by Transformation 3, as shown in Figure 3.

Lemma 3. If $K$ is obtained from $G$ by Transformation 3 as shown in Figure 3, then

$$
H M(K)>H M(G) .
$$

Proof. From Figure 3, let $d_{G_{1}}(u)=x$ and $d_{G_{2}}(v)=y$, while $w=u+v$ (merge $u$ and $v$ to obtain $w$ ) with $d_{K}(w)=x+y+$ $a-1$, where $a \geq 2$. If $a=2$, then

$$
\begin{aligned}
H M(K)-H M(G)> & (x+y+2-1+1)^{2} \\
& -(x+y+2)^{2}=0 .
\end{aligned}
$$

If $a \geq 3$, then

$$
\begin{aligned}
H M(K)-H M(G)> & (a-1)(x+y+a-1+1)^{2} \\
& -(x+3)^{2}-(y+3)^{2} \\
& +16(a-3) \\
= & (a-1)(x+y+a)^{2}-(x+3)^{2} \\
& -(y+3)^{2}-16(a-3) \\
> & (x+y+a)^{2}-(x+3)^{2} \\
& +(x+y+a)^{2}-(y+3)>0 .
\end{aligned}
$$

Transformation 4. Let $H$ be a nontrivial acyclic subgraph of $G$ with $|H|=t$ which is attached at $u_{1}$ in graph $G$; let $u$ and $v$ be two neighbors of $u_{1}$ different from those in $H$; also $d(u)=x$ and $d(v)=y$. If $K=G-\left(H-u_{1}\right)+u_{1} u_{2}+u_{2} u_{3}+\cdots+u_{t} v$, we say that $K$ is obtained from $G$ by Transformation 4 , as shown in Figure 4.

Lemma 4. Let $G$ and $K$ be two graphs, as shown in Figure 4. Then

$$
H M(G)>H M(K) .
$$

Proof. From Transformation 2, we know that $H M(G) \geq$ $H M\left(G_{1}\right)$. So, we only prove the following inequality:

$$
\begin{aligned}
H M\left(G_{1}\right)> & H M(K), \\
H M\left(G_{1}\right)-H M(K)= & \left(d_{G_{1}}\left(u_{t-1}\right)+d_{G_{1}}\left(u_{t}\right)\right)^{2} \\
& +\left(d_{G_{1}}\left(u_{1}\right)+d_{G_{1}}\left(u_{2}\right)\right)^{2} \\
& +\left(d_{G_{1}}(u)+d_{G_{1}}\left(u_{1}\right)\right)^{2} \\
& +\left(d_{G_{1}}(v)+d_{G_{1}}\left(u_{1}\right)\right)^{2} \\
& -\left(d_{K}\left(u_{t-1}\right)+d_{K}\left(u_{t}\right)\right)^{2} \\
& -\left(d_{K}\left(u_{1}\right)+d_{K}\left(u_{2}\right)\right)^{2} \\
& -\left(d_{K}(u)+d_{G_{1}}\left(u_{1}\right)\right)^{2} \\
& -\left(d_{K}(v)+d_{K}\left(u_{1}\right)\right)^{2} \\
= & (x+3)^{2}+(y+3)^{2}-(x+2)^{2} \\
& -(y+2)^{2}+2>0 .
\end{aligned}
$$

Therefore, the proof is complete.

Let $G$ be a nontrivial connected graph. Two vertices $u$ and $v$ are said to be equivalent if $G-u \cong G-v$. Clearly, $|N(u)|=|N(v)|$ and their neighbors have the same degree sequence.

Transformation 5. Let $G_{0}$ be a nontrivial connected graph and $u$ and $v$ are equivalent vertices in $G_{0}$ such that $d_{G_{0}}(u)=$ $d_{G}(v)=x$. Let $G$ be the graph obtained by attaching $S_{a+1}$ and $S_{b+1}$ at the vertices $u$ and $v$ of $G_{0}$, respectively, with $a \geq b \geq 1$. If $K$ is the graph obtained by deleting the $b$ pendant vertices at $v$ in $G$ and connecting them to $u$ of $G$, respectively, as shown in Figure 5 . We say that $K$ is obtained from $G$ by Transformation 5.

Lemma 5. If $K$ is obtained from $G$ by Transformation 5 as shown in Figure 5, then

$$
H M(G)<H M(K) .
$$




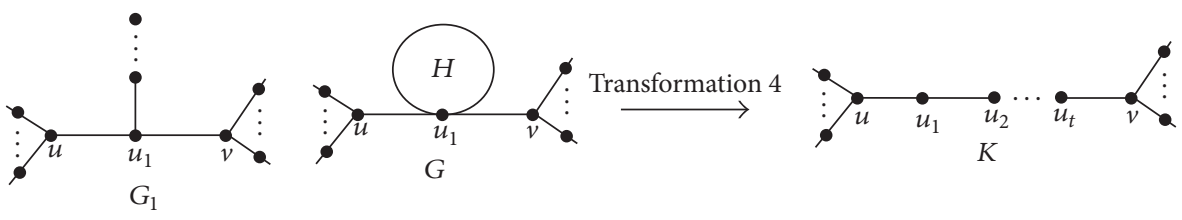

Figure 4: Transformation 4.

Proof. We have

$$
\begin{aligned}
H M(K)-H M(G)> & a\left(d_{K}(u)+1\right)^{2} \\
& +b\left(d_{K}(u)+1\right)^{2} \\
& -a\left(d_{G}(u)+1\right)^{2} \\
& -b\left(d_{G}(u)+1\right)^{2} \\
= & a(x+a+b+1)^{2} \\
& +b(x+a+b+1)^{2} \\
& -a(x+a+1)^{2} \\
& -b(x+b+1)^{2}>0 .
\end{aligned}
$$

\section{Main Results}

In this section, we characterized the extremal graph with respect to $H M(G)$ among acyclic, unicyclic, and bicyclic graphs. First, we will define some notations which will be used later. $B_{n}$ denotes the set of all connected bicyclic graphs with order $n$. Now we define three special classes of graphs. Let $P_{n}^{k, l, m}$ be the graph obtained by connecting two cycles $C_{k}$ and $C_{m}$ with a path $P_{l}$ with $k+l+m-2=n$. Let $C_{n}(p, q)$ be the graph which contains only two cycles $C_{p}$ and $C_{q}$ having a common vertex with $p+q-1=n$, and let $C_{n}(l, r, t)$ be the graph obtained by fusing two triples of pendant vertices of three paths $P_{l}, P_{r}$, and $P_{t}$ to two vertices with $l+r+t-4=n$, where $2 \leq l \leq r \leq t$ without loss of generality. Let $G$ be a bicyclic graph containing one of the three graphs $P_{n}^{k, l, m}$, $C_{n}(p, q)$, and $C_{n}(l, r, t)$ as its subgraph; then we will call it a brace of $G$. We set $B_{n}^{1}, B_{n}^{2}$, and $B_{n}^{3}$ to be the set of all bicyclic graphs which include $P_{n}^{k, l, m}, C_{n}(p, q)$, and $C_{n}(l, r, t)$ as their brace, respectively. Clearly, $B_{n}^{1}, B_{n}^{2}$, and $B_{n}^{3}$ are the partitioned subsets of $B_{n}$.

If $G$ is an acyclic graph with order $n$, then, by Lemmas 1 and 2 , the following result holds.

Theorem 6. Let $G$ be an acyclic graph with order $n$. Then

$$
16 n-30 \leq H M(G) \leq n^{2}(n-1),
$$

where the lower bound is achieved if and only if $G \cong P_{n}$ and the upper bound is achieved if and only if $G \cong S_{n}$.

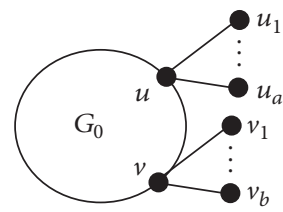

G

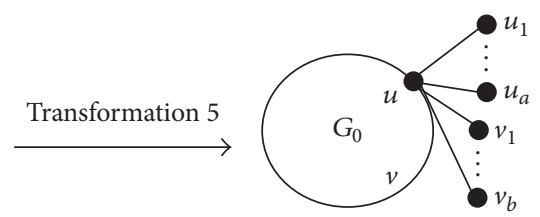

K
Figure 5: Transformation 5.

Theorem 7. Let $G$ be a unicyclic graph with $n$ vertices. Then

$$
H M\left(C_{n}\right) \leq H M(G) \leq H M\left(S_{n}^{1}\right),
$$

where the lower bound is achieved if and only if $G \cong C_{n}$ and the upper bound is achieved if and only if $G \cong S_{n}^{1}$.

Proof. Being a unicyclic graph $G$ contains a unique cycle $C_{l}$. By Lemma 3, we can obtain the graph $K$ in which the length of the cycle is three and its $H M$ is increased strictly and then, by using Lemma 5 , we can get the uniquely maximum graph $S_{n}^{1}$ with respect to $H M$ (Figure 6). By using Lemmas 1 and 4, we find that the minimum graph is $C_{n}$.

Theorem 8. Let $G$ be a bicyclic graph with $n$ vertices. Then

$$
16 n+70 \leq H M(G) \leq n^{3}-n^{2}+8 n+56,
$$

where the lower bound is achieved if and only if $G \in\left\{P_{n}^{k, l, m}\right.$ : $l \geq 3\} \cup\left\{C_{n}(r, l, t): l \geq 3\right\}$ and the upper bound is achieved if and only if $G \cong S_{n}^{2}$.

Proof. By simple calculation, one can obtain $\operatorname{HM}\left(S_{n}^{2}\right)=n^{3}-$ $5 n^{2}+16 n+4$. So, we show that if $G \neq S_{n}^{2}$, then $H M(G)<$ $H M\left(S_{n}^{2}\right)$.

Case 1 ( $G$ Contains $K_{4}-e$ as Its Brace). As $G$ contains $K_{4}-e$ as its brace, by using Lemmas 2 and 5, we can obtain a new bicyclic graph $G^{1}$ whose $H M$ is more than that of $G$ (see Figure 6). Clearly, $H M\left(G^{1}\right)<n^{3}-5 n^{2}+16 n+4$.

Case $2\left(K_{4}-e\right.$ Is Not the Brace of $\left.G\right)$. Though $G$ does not contain the subgraph $K_{4}-e$, by Lemma 3, maybe there is 


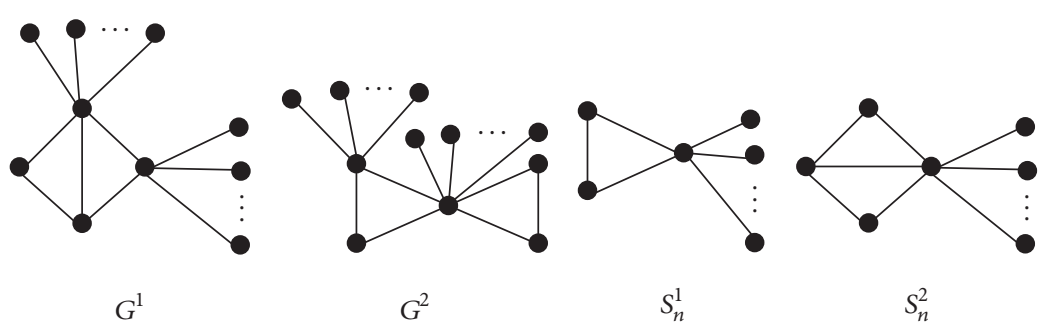

FIgURE 6: Some graphs.

a bicyclic graph having brace $K_{4}-e$ whose $H M$ is greater than $G$. So we have two subcases.

Subcase $2.1\left(C_{n}(3,2, m)\right.$ Is the Brace of $\left.G\right)$. By Lemma 3, Subcase 2.1 is converted to Case 1 .

Subcase $2.2\left(S_{n}(3,2, m)\right.$ Is Not the Brace of $\left.G\right)$. By Lemmas 2,3 , and 5 , we get a new bicyclic graph $G^{2}$ (Figure 6) whose $H M$ is more than that of $G$. It is easy to verify that $H M\left(G^{2}\right)<$ $n^{3}-5 n^{2}+16 n+4$.

Now we obtain the lower bound of bicyclic graphs with respect to $H M$. From Lemmas 1, 2, and 4, we find that the extremal graph of the minimum $H M$ in bicyclic graphs must be element from the set $\left\{P_{n}^{k, l, m}, C_{n}(p, q), C_{n}(r, l, t)\right\}$.

Clearly, $H M\left(P_{n}^{k, l, m}\right)=16 n+70, H M\left(C_{n}(p, q)\right)=16 n+96$, and $H M\left(C_{n}(r, l, t)\right)=16 \mathrm{n}+70$.

So, we check the lower bound and equality holds if and only if $G \in\left\{P_{n}^{k, l, m}: l \geq 3\right\} \cup\left\{C_{n}(r, l, t): l \geq 3\right\}$.

\section{Competing Interests}

The authors declare that there are no competing interests regarding the publication of this paper.

\section{Acknowledgments}

This work was supported in part by the State Grid Anhui Economic Research Institute (no. 1P12001500010681000000) and Natural Science Foundation of Anhui Province of China (nos. KJ2015A331 and KJ2013B105).

\section{References}

[1] H. Wiener, "Structural determination of paraffin boiling points," Journal of the American Chemical Society, vol. 69, no. 1, pp. 1720, 1947.

[2] I. Gutman and N. Trinajstić, "Graph theory and molecular orbitals, total $\pi$-electron energy of alternant hydrocarbons," Chemical Physics Letters, vol. 17, pp. 535-538, 1972.

[3] K. C. Das, K. Xu, and I. Gutman, "On Zagreb and Harary indices," MATCH. Communications in Mathematical and in Computer Chemistry, vol. 70, no. 1, pp. 301-314, 2013.

[4] C. M. da Fonseca and D. Stevanovic, "Further properties of the second Zagreb index," MATCH. Communications in Mathematical and in Computer Chemistry, vol. 72, no. 3, pp. 655-668, 2014.
[5] I. Gutman, M. K. Jamil, and N. Akhter, "Graphs with fixed number of pendent vertices and minimal first Zagreb index," Transactions on Combinatorics, vol. 4, no. 1, pp. 43-48, 2015.

[6] J. Estes and B. Wei, "Sharp bounds of the Zagreb indices of $k$ -trees," Journal of Combinatorial Optimization, vol. 27, no. 2, pp. 271-291, 2014.

[7] S. A. U. H. Bokhary, M. Imran, and S. Manzoor, "On molecular topological properties of dendrimers," Canadian Journal of Chemistry, vol. 94, no. 2, pp. 120-125, 2015.

[8] M. Imran, A. Q. Baig, and H. Ali, "On topological properties of dominating David derived networks," Canadian Journal of Chemistry, vol. 94, no. 2, pp. 137-148, 2015.

[9] A. Miličević, S. Nikolić, and N. Trinajstić, "On reformulated Zagreb indices," Molecular Diversity, vol. 8, no. 4, pp. 393-399, 2004.

[10] N. De, "Some bounds of reformulated Zagreb indices," Applied Mathematical Sciences, vol. 6, no. 101-104, pp. 5005-5012, 2012.

[11] A. Ilić and B. Zhou, "On reformulated Zagreb indices," Discrete Applied Mathematics, vol. 160, no. 3, pp. 204-209, 2012.

[12] S. Ji, X. Li, and B. Huo, "On reformulated Zagreb indices with respect to acyclic, unicyclic and bicyclic graphs," MATCH Communications in Mathematical and in Computer Chemistry, vol. 72, no. 3, pp. 723-732, 2014.

[13] G. H. Shirdel, H. RezaPour, and A. M. Sayadi, "The hyperzagreb index of graph operations," Iranian Journal of Mathematical Chemistry, vol. 4, no. 2, pp. 213-220, 2013.

[14] S. Wang and B. Wei, "Multiplicative Zagreb indices of k-trees," Discrete Applied Mathematics, vol. 180, pp. 168-175, 2015.

[15] W. Gao and W. Wang, "The eccentric connectivity polynomial of two classes of nanotubes," Chaos, Solitons \& Fractals, vol. 89, pp. 290-294, 2016.

[16] W. Gao and W. F. Wang, "The fifth geometric-arithmetic index of bridge graph and carbon nanocones," Journal of Difference Equations and Applications, 2016.

[17] J.-B. Liu and X.-F. Pan, "Asymptotic incidence energy of lattices," Physica A. Statistical Mechanics and its Applications, vol. 422, pp. 193-202, 2015.

[18] J.-B. Liu, X.-F. Pan, F.-T. Hu, and F.-F. Hu, "Asymptotic Laplacian-energy-like invariant of lattices," Applied Mathematics and Computation, vol. 253, pp. 205-214, 2015.

[19] J. A. Bondy and U. S. Murty, Graph Theory, vol. 244 of Graduate Texts in Mathematics, Springer, Berlin, Germany, 2008. 


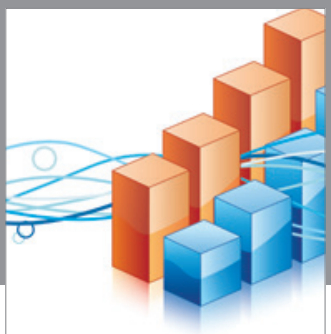

Advances in

Operations Research

vatem alat4

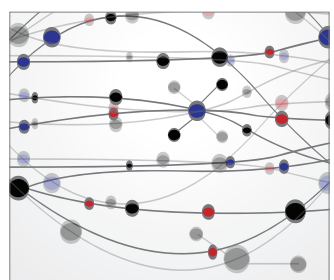

\section{The Scientific} World Journal
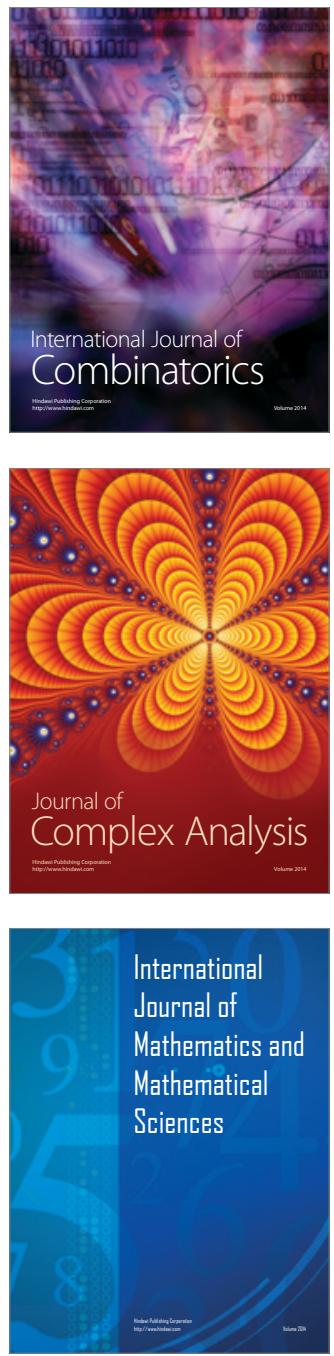
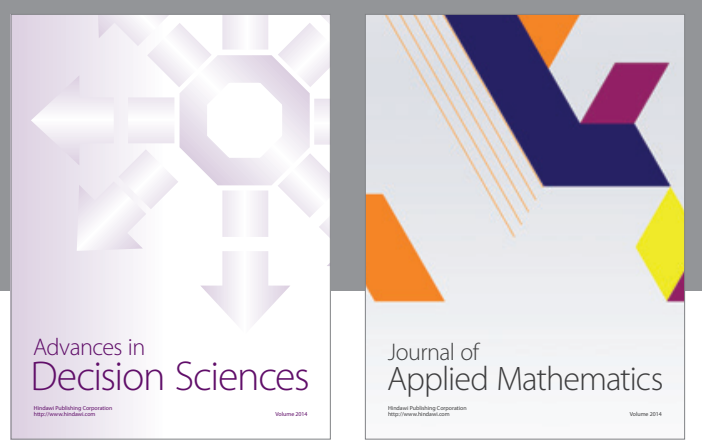

Algebra

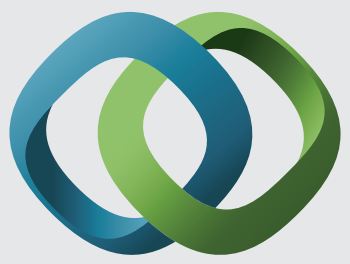

\section{Hindawi}

Submit your manuscripts at

https://www.hindawi.com
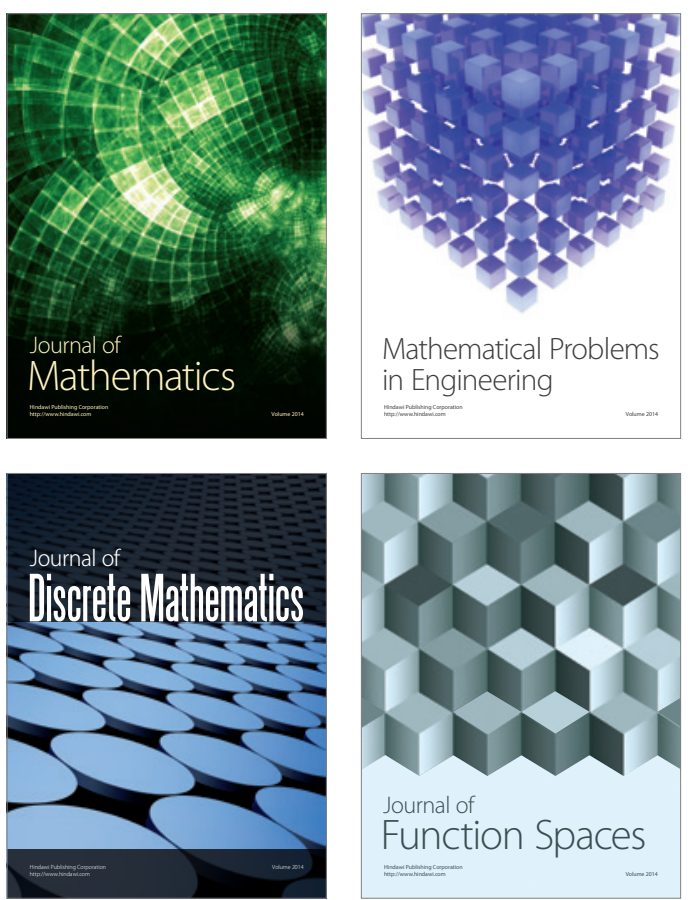

Mathematical Problems in Engineering
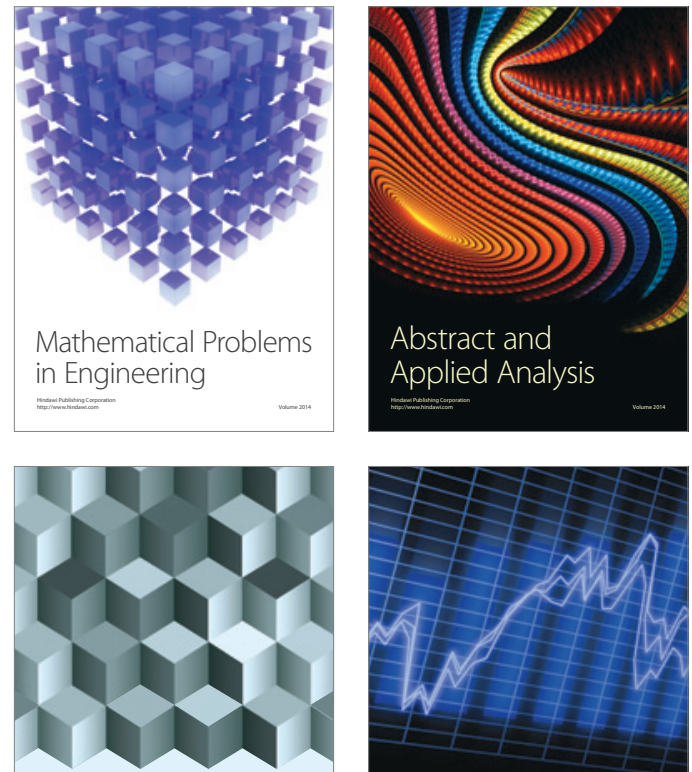

Journal of

Function Spaces

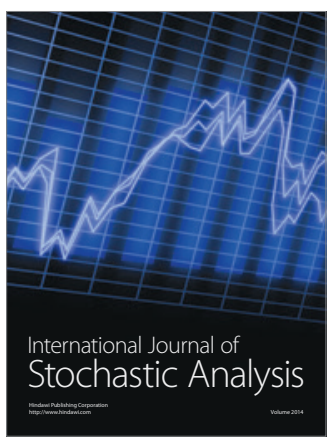

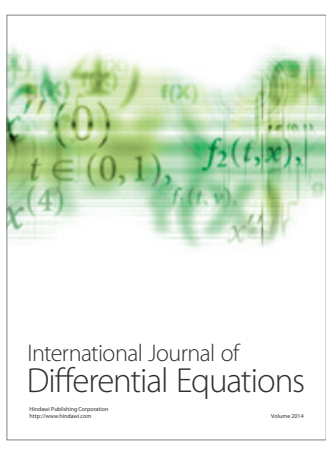
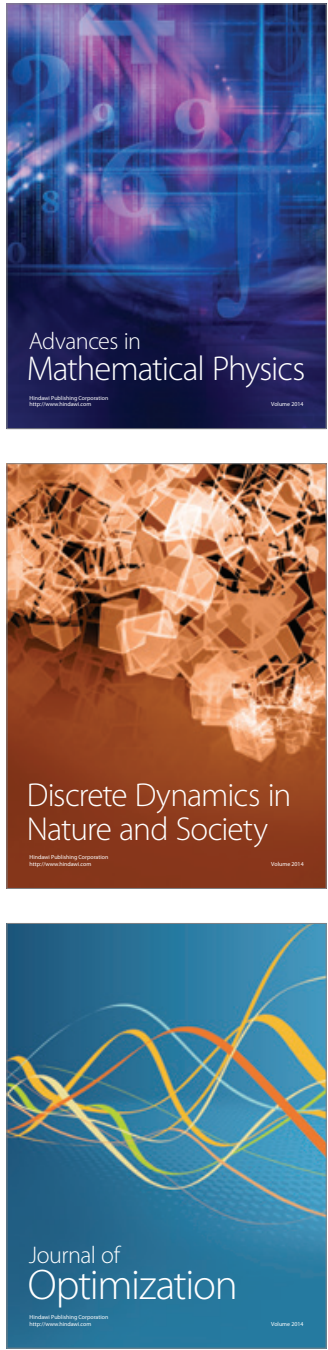